\title{
Cowden-Syndrom (Multiples Hamartom-Syndrom)
}

\section{Cowden Syndrome (Multiple Hamartoma Syndrome)}

Autoren

Institut

\author{
N. Scola, T. Gambichler
}

Klinik für Dermatologie und Allergologie der Ruhr-Universität Bochum (Direktor: Prof. Dr. P. Altmeyer)

\section{Bibliografie}

DOI $10.1055 / \mathrm{s}-2008-1077637$

Akt Dermatol 2008; 34:

433-436 @ Georg Thieme

Verlag KG Stuttgart · New York ISSN 0340-2541

Korrespondenzadresse Nina Scola, Assistenzärztin Klinik für Dermatologie und Allergologie der Ruhr-Universität-Bochum Gudrunstraße 56 44791 Bochum nina.scola@web.de

\section{Zusammenfassung \\ $\nabla$}

Das Cowden-Syndrom ist ein Hamartom-Symptomkomplex mit individuell unterschiedlicher klinischer Ausprägung. Pathognomonische dermatologische Läsionen sind faziale mukokutane Papillome, faziale Tricholemmome und akrale Keratosis. Von größter prognostischer und therapeutischer Bedeutung ist das Auftreten von Mali-

\section{Einleitung \\ $\nabla$}

Das Cowden-Syndrom wurde erstmalig von Lloyd und Dennis 1963 beschrieben und nach der ersten Patientin benannt [1]. Charakteristisch ist das Auftreten von bestimmten Hautveränderungen, Schilddrüsenerkrankungen und Mammopathien sowie das erhöhte Risiko der Malignomentstehung. Histologisch finden sich $\mathrm{Ha}-$ martome sowohl der Haut als auch des Gastrointestinaltraktes. Es konnte ein defektes Tumorsuppressorgen als Ursache des Cowden-Syndroms identifiziert werden. Wir berichten nachfolgend über eine 56-jährige Patientin mit einem Schilddrüsenkarzinom in der Anamnese sowie charakteristischen HPV-negativen Fibropapillomen der Unterlippe und Mundschleimhaut.

\section{Fallbericht \\ $\nabla$ \\ Anamnese}

Eine 56-jährige Patientin bemerkte seit mindestens zehn Jahren warzenartige Hautveränderungen mit sukzessivem Größenwachstum an der Unterlippe. Die Hautveränderungen verursachten keine Beschwerden. Bei der Patientin war vor über 10 Jahren ein Schilddrüsenkarzinom festgestellt und konsekutiv eine Thyreoidektomie durchgeführt worden. An weiteren Nebendiagnosen bestanden ein metabolisches Syndrom gnomen, insbesondere Mamma- und Schilddrüsenkarzinomen. Die Diagnose wird anhand von klinischen Kriterien gestellt. Aufgrund des erhöhten Risikos sind Krebsvorsorgeuntersuchungen unerlässlich. Wir berichten über eine Patientin mit Cowden-Syndrom einschließlich fazialer Papillome, Schilddrüsenkarzinom und intestinaler hamartöser Polyposis.

mit Diabetes mellitus Typ II NIDDM, Adipositas und arteriellem Hypertonus. Die Familienanamnese war in Bezug auf Haut- und Erbkrankheiten leer.

\section{Hautbefund}

Es zeigten sich an der linken Hälfte der Unterlippe (Außenseite und enoral) Beete von verrukösen, zerklüfteten Papeln. An der buccalen Mundschleimhaut fanden sich beiderseits vereinzelte Papeln ( $\bullet$ Abb. 1 u. 2). Nebenbefundlich fielen eine ausgeprägte Lingua plicata ( $\bullet$ Abb. 2) und axillär beidseits eine Akanthosis nigricans mit ausgedehnter Papillomatose auf ( $\bullet$ Abb. 3).

\section{Histologie}

Zur Diagnosesicherung führten wir Probeexzisionen an Lippen- und Wangenschleimhaut durch, deren histologische Begutachtung Fibropapillome zeigten. Die Präparate wiesen eine korbgeflechtartige Orthokeratose, geringgradige Akanthose, lockeres koriales Bindegewebe, vermehrte Anschnitte von dilatierten kapillären Blutgefäßen mit geringgradig entzündlichen Infiltraten aus Lymphozyten und Histiozyten perivaskular auf. Stellenweise lag eine kompakte Parakeratose vor. Es fanden sich Einlagerungen eines zellreichen Entzündungsexsudates mit zahlreichen Neutrophilen. In der PAS-Spezialfärbung fand sich kein Nachweis von Pilzelementen. Jegliche Adnexstrukturen fehlten. Die Probe einer Papel 


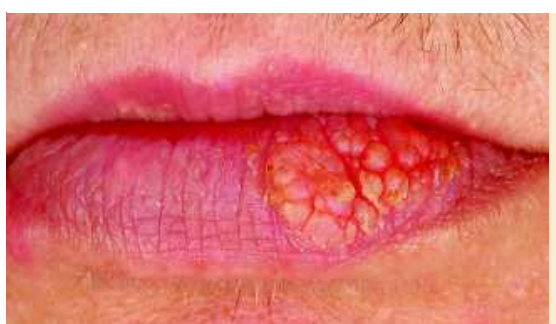

Abb. 1 Beetartige, verruziforme Papeln der Unterlippe.
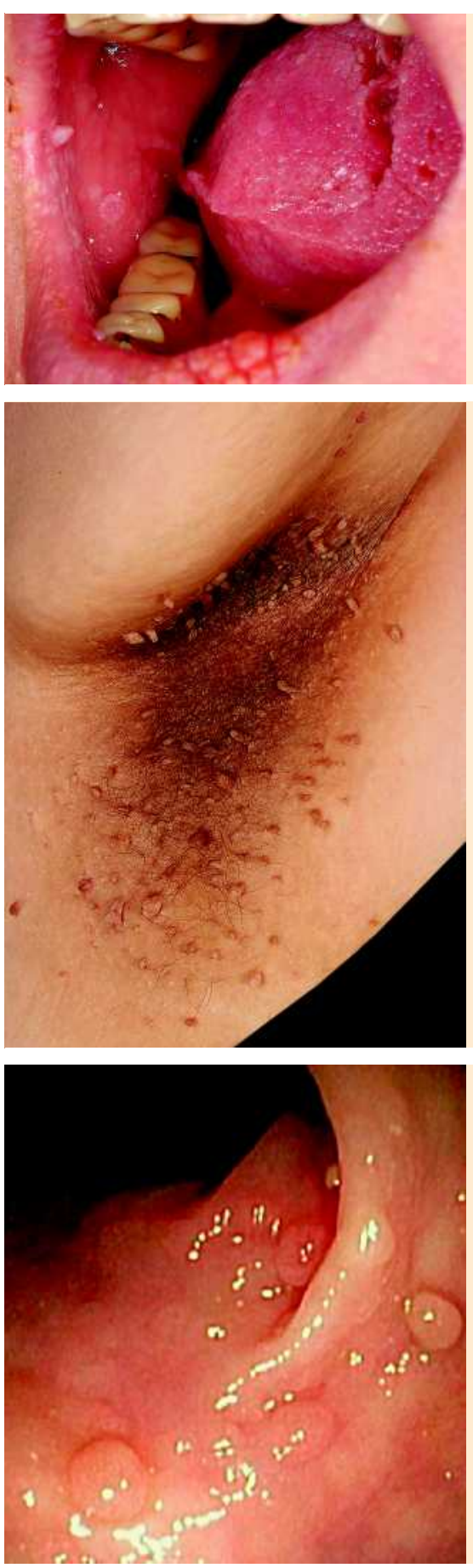

Abb. 4 Koloskopie mit Nachweis mehrerer Polypknospen.

der Wangenschleimhaut wurde mittels PCR auf HPV-DNA untersucht - das Ergebnis war negativ.

2 Mehrere Papeln der Mundschleimhaut buccal sowie ausgeprägte Lingua plicata.

Abb. 3 Acanthosis nigricans sowie multiple pendulierende Fibrome.

\section{Koloskopie}

In der rechten Flexur zeigte sich ein ca. $10 \mathrm{~mm}$ großer Polyp. Im gesamten Kolon mit Betonung im Rektum stellten sich multiple, mehr oder weniger große Polypknospen dar ( $\bullet$ Abb. 4).

\section{Labor}

Unauffällig bzw. altersentsprechend waren Differenzialblutbild, Leber-/Nierenparameter, Blutzucker, CRP, die Tumormarker CEA, CA15-3, CA 19-9 und CEA.

\section{Diagnose, Therapie und Verlauf}

Die klinischen Befunde und das Schilddrüsenkarzinom in der Eigenanamnese waren gut vereinbar mit einem Cowden-Syndrom. Die Diagnose stellten wir anhand der modifizierten Kriterien nach Salem und Steck [20,23]. Die Fibropapillome der Unterlippe wurden mittels Laserablation entfernt. Röntgen des Thorax und Sonografie des Abdomens zeigten keinen Hinweis für Malignität. Einer neurologischen Untersuchung sowie einer kranialen Computertomografie wollte sich die Patientin nicht unterziehen. Wir stellten die Patientin zur genetischen Familienberatung vor und empfahlen regelmäßige Krebsvorsorgen, insbesondere auf dem gynäkologischen Fachgebiet.

\section{Besprechung}

Beim Cowden-Syndrom handelt es sich um einen Symptomkomplex mit individuell unterschiedlicher klinischer Ausprägung [2]. Als histologische Gemeinsamkeit der Veränderungen lassen sich Hamartome nachweisen, die allen drei Keimblättern entspringen können, weshalb das Cowden-Syndrom auch als Multiples Hamartom-Syndrom bezeichnet wird [3]. Führende Symptome sind charakteristische Hautveränderungen, Mammound Thyreoidopathien. Bei $80 \%$ der Patienten treten pathognomonische oder weniger spezifische Hautveränderungen auf [4]. Die charakteristischen Hautveränderungen werden als mukokutane gefurchte Papeln im Gesichtsbereich, vor allem periorbital und enoral (wie im dargestellten Fallbeispiel), akrale papulöse Keratosis an Hand- und Fußrücken und Tricholemmome und Trichoepitheliome im Gesichtsbereich definiert [5]. An weniger spezifischen Hautveränderungen finden sich gehäuft die Lingua plicata, Lipome, Xanthome, Café-au-lait-Flecken, Vitiligo und andere Läsionen [6,7].

Die häufigsten extrakutanen Manifestation des Cowden-Syndroms sind Thyreoidopathien bei ca. $2 / 3$ der Betroffenen, wie z. B. follikuläre Adenome [8], sowie Mammopathien bei ca. der Hälfte der Patienten vorkommend [9]. Auch intestinale, meist asymptomatische Hamartome [10], treten auf und sind zumeist in Kolon und Rektum lokalisiert [11]. Histologische Kennzeichen der intestinalen Hamartome sind eine hohe Anzahl von normotypischen Drüsen mit mukoidem Inhalt, ödematöse und entzündliche Veränderungen der Lamina propria und lymphoplasmazelluläre Infiltration [12]. Ebenso wurden neurologische Veränderungen beobachtet (z. B. Makrozephalien und das Lhermitte-Duclos-Syndrom). Letzteres ist ein seltenes, benignes dysplastisches zerebelläres Gangliozytom, das beim Cowden-Syndrom öfter beobachtet wird [13]. Auch otolaryngologische, opththalmologische, gynäkologische, renale und kraniomaxillofaziale Manifestationen sind beschrieben worden [14]. Besondere Bedeutung für die Prognose hat die hohe Prävalenz von Karzinomen. Bei $1 / 3$ der Patienten treten Mamma-Karzinome zumeist beidseitig auf. Bei immerhin $12 \%$ der Patienten werden 


\section{Pathognomonische Läsionen}

Faziale Tricholemmome

Akrale Keratose

Papillomatöse Papeln

Mukosale Läsionen

\section{Major-Kriterien}

Mammakarzinom

Thyreoidkarzinom (meist follikulär)

Endometriumkarzinom

Lhermitte-Duclos-Syndrom

Makrozephalie

\section{Minor-Kriterien}

Andere thyroidale Erkrankungen

Mentale Retardierung

Intestinale hamartomatöse Polypen

Fibrozystische Mastopathie

Lipome

Fibrome

Urogenitale Tumoren

Urogenitale Malformationen

Lingua plicata
Tab. 1 Diagnostische Krite-

rien für das Cowden-Syndrom.
Schilddrüsenkarzinome beobachtet $[15,16]$. Auch Tumore des Gastrointestinaltraktes, Nierenkarzinome, Ovarial-Karzinome, Endometriumkarzinome und Hauttumore (z.B. Merkelzell-Karzinom) werden gehäuft beobachtet [14].

Es sind fast nur Kaukasier (95\%) und überwiegend (60\%) Frauen betroffen [17]. Zumeist manifestiert sich die Erkrankung in der 3. oder 4. Lebensdekade [16]. Obgleich ein autosomal-dominanter Erbgang vorliegt, findet sich nur in $10-15 \%$ der Fälle eine familiäre Häufung [12]. Die Prävalenz wurde vor Identifikation des Gendefektes zunächst auf 1:1000000 geschätzt und aktuell mit $1: 200000$ angegeben, liegt jedoch wahrscheinlich noch höher $[3,18,19]$. Zugrunde liegt ein defektes PTEN-Gen auf 10q23.3 („Phosphatase und Tensin Homolog-Gen“), das als Tumorsuppressorgen fungiert [20]. Das Genprodukt ist eine PI3-Phosphatase, welche die Zellproliferation kontrolliert. Ein Verlust der PTEN-Genfunktion kann bei vielen menschlichen Karzinomarten gefunden werden [21]. Derselbe Defekt liegt auch bei der juvenilen Polyposis des Darms und beim Bannayan-Riley-Ruvalcaba-Syndrom vor, einer ebenfalls autosomal-dominant vererbten Genodermatose [20,22]. Das Cowden-Syndrom und das Bannayan-Riley-Ruvalcaba-Syndrom weisen eine teilweise überlappende Symptomatik auf: Die klassischen Triade des BannayanRiley-Ruvalcaba-Syndroms besteht aus Makrozephalie, genitaler Lentiginose und - analog zum Cowden-Syndrom - intestinaler Polyposis [23]. Auch verruca-ähnliche Hautveränderungen im Gesichtsbereich sind bei beiden Syndromen beschrieben worden [23].

Die Diagnosestellung des Cowden-Syndroms erfolgt modifiziert nach Salem und Steck [20,24] anhand von klinischen Kriterien. Es werden pathognomonische Hautläsionen, Major-Kriterien und Minor-Kriterien differenziert ( $\bullet$ Tab. 1). Die Diagnose kann bei folgenden Konstellationen gestellt werden:

1. Vorhandensein von pathognomonischen Läsionen:

a) mindestens 6 faziale Papeln, davon mindestens 3 Tricholemmome

b) faziale Papeln und enorale mukosale Papillomatose

c) enorale mukosale Papillomatose und akrale Keratose

d) mindestens 6 palmoplantare keratotische Läsione

2. Vorhandensein zweier Major-Kriterien, davon eines Makrozephalie oder Lhermitte-Duclos-Syndrom

3. Vorhandensein eines Major-Kriteriums und dreier MinorKriterien

4. Vorhandensein von vier Minorkriterien

Bei direkten Verwandten eines betroffenen Patienten kann die Diagnose bereits gestellt werden bei einer der folgenden drei Konstellationenen:

1. Vorhandensein von mukokutanen pathognomonischen Läsionen

2. Vorhandensein eines Major-Kriteriums

3. Vorhandensein von zwei Minor-Kriterien
Aufgrund der hohen Prävalenz von Malignomen kommen gründlichen Vorsorgeuntersuchungen eine besonders große Bedeutung zu. Empfohlen werden jährliche gynäkologische Vorsorgeuntersuchungen, zumindest initial Schilddrüsenuntersuchungen und initial dermatologische Vorsorgeuntersuchungen sowie Koloskopien. Des Weiteren besteht die Möglichkeit, genetische Beratung und Familienuntersuchungen durchzuführen.

\section{Abstract}

\section{Cowden Syndrome (Multiple Hamartoma Syndrome) $\nabla$}

The Cowden syndrome is a complex hamartomous symptom with individually varying clinical manifestations. Diagnostic dermatological lesions include facial mucocutaneous papillomas, facial trichilemmoma and acral keratosis. Most important for prognostic and therapeutic reasons is the increased incidence of malignomas in Cowden-Syndrom such as breast and thyreoid cancer. The diagnosis of Cowden syndrome is based on clinical criteria. Due to the higher risk of cancer development in patients with Cowden syndrome, it is very important to perform complete medical follow-ups on a regular basis. We report a patient with Cowden syndrome including facial papillomatosis, thyreoid cancer and intestinal polyposis.

\section{Literatur}

1 Lloyd K, Dennis $M$. Cowden's disease: a possible new symptom complex with multiple system involvement. Ann Int Med 1963; 58: 136 142

2 Boardman LA. Heritable colorectal cancer syndromes: recognition and preventive management. Gastroenterol Clin North Am 2002; 31: $1107-1131$

3 Schreibman IR, Baker M, Amos C, McGarrity TJ. The hamartomatous polyposis syndromes: a clinical and molecular review. Am J Gastroenterol 2005; 100: 476 - 490

4 Starink TM, van der Veen JP, Arwert F, de Waal LP, de Lange GG, Gille JJ, Eriksson AW. The Cowden syndrome: a clinical and genetic study in 21 patients. Clin Genet 1986; 29: 222-233

5 Hand JL, Rogers RS 3rd. Oral manifestations of genodermatoses. Dermatol Clin 2003; 21: 183-194

6 Thyresson HN, Doyle JA. Cowden's disease (multiple hamartoma syndrome). Mayo Clin Proc 1981; 56: 179- 184

7 Scheper MA, Nikitakis NG, Sarlani E, SaukJJ, Meiller TF. Cowden syndrome: report of a case with immunohistochemical analysis and review of the literature. Oral Surg Oral Med Oral Pathol Oral Radiol Endod 2006; 101: $625-631$

8 Hemmings CT. Thyroid pathology in four patients with Cowden's disease. Pathology 2003; 35: 311 - 314

9 Starink TM. Cowden's disease: analysis of fourteen new cases. J Am Acad Dermatol 1984; 11: 1127-1141 
10 Marra G, Armelao F, Vecchio FM, Percesepe A, Anti M. Cowden's disease with extensive gastrointestinal polyposis. J Clin Gastroenterol 1994; 18: $42 \mathrm{ff}$.

11 Longy M, Lacombe D. Cowden disease. Report of a family and review. Ann Genet 1996; 39: 35-42

12 Carlson GJ, Nivatvongs S, Snover DC. Colorectal polyps in Cowden's disease (multiple hamartoma syndrome). Am J Surg Pathol 1984; 8: $763-770$

13 Nowak DA, Trost HA. Lhermitte-Duclos disease (dysplastic cerebellar gangliocytoma): a malformation, hamartoma or neoplasm? Acta Neurol Scand 2002; 105: 137- 145

14 Capitán Cañadas LM, Salinas Sánchez JL, Martínez Castillo SL, Labrot Moleón IL, Durán Moreno D, Sánchez López D, Valencia Laseca E. Multiple oral fibropapillomatosis as an initial manifestation of Cowden Syndrome. Case report. Med Oral Patol Oral Cir Bucal 2006; 11: E319E324

15 Ball S, Arolker M, Purushotham AD. Breast cancer, Cowden disease and PTEN-MATCHS syndrome. Eur J Surg Oncol 2001; 27: 604 -606

16 Salem OS, Steck WD. Cowden's disease (multiple hamartoma and neoplasia syndrome). A case report and review of the English literature. J Am Acad Dermatol 1983; 8: 686 - 696

17 Botma M, Russell DI, Kell RA. Cowden's disease: a rare cause of oral papillomatosis. J Laryngol Otol 2002; 116: 221 -223
18 Nelen MR, Padberg GW, Peeters EA, Lin AY, van den Helm B, Frants RR, Coulon V, Goldstein AM, van Reen MM, Easton DF, Eeles RA, Hodgsen $S$, Mulvihill JJ, Murday VA, Tucker MA, Mariman EC, Starink TM, Ponder BA, Ropers HH, Kremer H, Longy M, Eng C. Localization of the gene for Cowden disease to chromosome 10q22-23. Nat Genet 1996; 13: $114-116$

19 Segura Saint-Gerons R, Ceballos Salobreña A, Toro Rojas M, Gándara Rey JM. Oral manifestations of Cowden's disease. Presentation of a clinical case. Med Oral Patol Oral Cir Bucal 2006; 11: E421 - E424

20 Frayling IM, Bodmer WF, Tomlinson IP. Allele loss in colorectal cancer at the Cowden disease/juvenile polyposis locus on 10q. Cancer Genet Cytogenet 1997; 97: 64-69

21 Leslie NR, Downes CP. PTEN function: how normal cells control it and tumour cells lose it. Biochem J 2004; $15: 382$ (Pt 1): 1 - 11

22 Zigman AF, Lavine JE, Jones MC, Boland CR, Carethers JM. Localization of the Bannayan-Riley-Ruvalcaba syndrome gene to chromosome 10q23. Gastroenterology 1997; 113: 1433-1437

23 Erkek E, Hizel S, Sanlý C, Erkek AB, Tombakoglu M, Bozdogan O, Ulkatan $S$, Akarsu C. Clinical and histopathological findings in Bannayan-RileyRuvalcaba syndrome. J Am Acad Dermatol 2005; 53: 639-643

24 Eng $C$. Will the real Cowden syndrome please stand up: revised diagnostic criteria. J Med Genet 2000; 37: 828 - 830 